Proc. of The Fifth Intl. Conf. On Advances in Applied Science and Environmental Engineering - ASEE 2016

Copyright (C) Institute of Research Engineers and Doctors, USA .All rights reserved.

ISBN: 978-1-63248-086-6 doi: 10.15224/ 978-1-63248-086-6-54

\title{
Transgenic Natural Killer Cells
}

\author{
Alireza Pirahmadian (PhD Student, Genetics And Molecular Biology), Dr.Shahrudin Abdul Razak (PhD Molecular Genetics) \\ ,Institute of biological science, Faculty Of Science ,University Of Malaya(50603), Kuala lumpur ,Malaysia.
}

\section{Abstract}

Adaptive immunity, with its rearranging immunoglobulin and T-cell receptors, has caught most of the attention of current genetic and immunological research and overshadowed the importance of the receptors expressed by cells of the innate immune system. Natural Killer cells have evolved two main receptor systems to carry out their functions, both of them involving activating and inhibitory receptors include members from the Immunoglobulin-like superfamily as well as lectinlike receptors. Killer Immunoglobulin-like receptors (KIR) are polymorphic cell surface molecules present on Natural Killer (NK) cells which recognize classical HLA class I molecules and in doing so provide an alternative means of modulating the immune response to infected or tumoural cells. In this study we did the Separation of Natural killer cells (NK cells) from rat's blood with the technique of LS column with the help of CD49 markers and Cultured of harvested NK cells in animal cells media (Dulbecco's Modified Eagle's Medium) with the help of animal cell culture technique. after pluralization of NK cells that was the Extraction of certain genes m RNA (Klra4,Klra8,KLRD1) of NK cells with the help of mRNA extraction technique .on the other hand we had to harvest hematopoietic stem cells from the thigh bone marrow of rats and culture them in to the special designed culture media for culturing stem cells (that would prevent the cell to form mature).at the end the main transfection would be done by transferring of protein synthesis system of Nk cells in to the harvested stem cells by the help of Lipofectamine .

Keywords- Natural killer cells, Killer immunoglobulin-like receptors, human leukocyte antigen, major histocompatibility complex class I, Dulbecco's Modified Eagle's Medium.

\section{Introduction:}

\section{Classification of immune system to innate and acquired immune responses}

The immunity system is responsible for response to an invading organism or immunogen which is received by our body [1]. This response is directed by innate and adaptive arms of the immune system. The border between adaptive and innate immune system in some aspects is not clear [1] [31]. In fact, these two systems are combined together in many responses. The innate immune system is mediated by the cells and factors which are responsible for the fast and first defense against infection whereas the adaptive immune response which is mainly organized by $\mathrm{T}$ and $\mathrm{B}$ cells are in charge for a long term memory response [2]. The cells involved in innate immunity are including different variety of myeloid and lymphoid cells such as neutrophils, basophils, macrophages and eosinophils [2][3]. The other cells like dendritic cells play a dual role in both - adaptive and innate immunity [5]. In recent years there are several reports about the role of natural killer (NK) cells in acquired immunity. Previously it was believed that NK cells are involved in innate immunity but recent reports have indicated that NK cells have memory against pathogens and after a primary infection [7], they respond faster in the second time. This response happens specially after viral infection [6][7]. The line and situation of factors and cytokines in innate and adaptive immunity is more complicated and there are lots of reports about the role of different cytokines in both immunities [8].

\section{Development of lymphoid lineages}

Blood cells are originated from the CD34+ pluripotent stem cells and developed from hematopoietic stem cells (HSCs). They are classified into two different main groups called lymphoid and myeloid lineages. Lymphoid lineage cells are including $\mathrm{T}$ and $\mathrm{B}$ lymphocytes as well as natural killer (NK) cells, while other blood cells such as erythrocytes, megakaryocytes, granulocytes and macrophages belong to the myeloid lineage [9] [11]. Dendritic cell is not clearly categorized because it is originated from both lymphoid and myeloid progenitors [10].

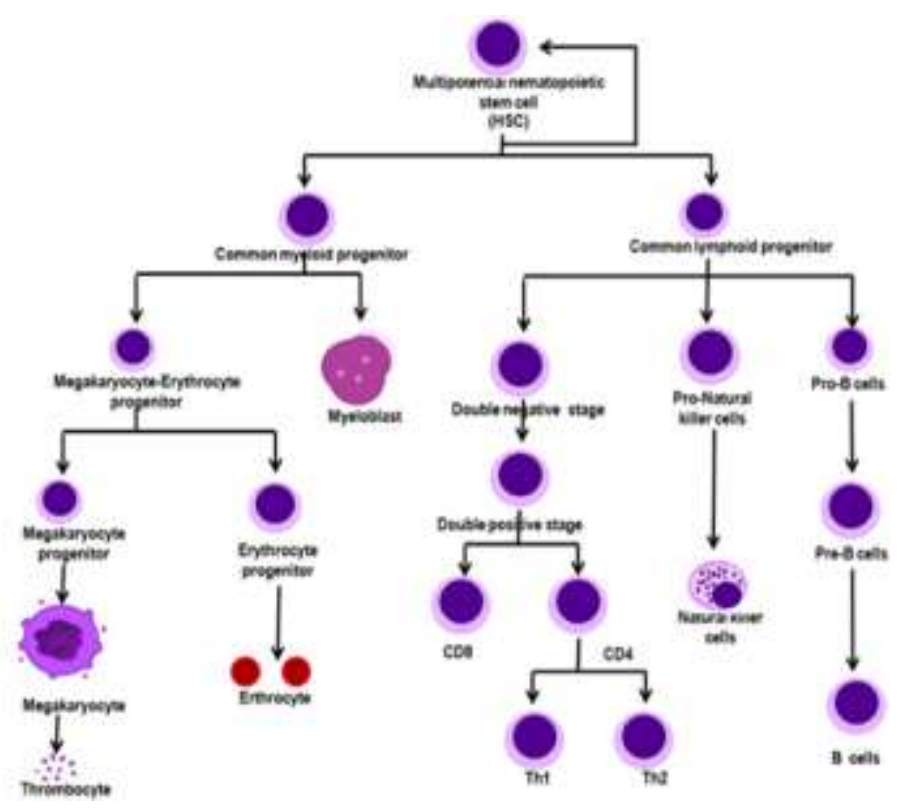

Figure 1. The differentiation and development of myeloid and lymphoid lineages (He et al., 2014).

\section{The structure of rat NK cells receptor families}

NK cells are defined with a group of receptors that can either activate or inhibit NK cell reactivity [15]. Activating receptors consists of receptors with interaction with soluble ligands like cytokines as well as receptors that react with cell surface 
Proc. of The Fifth Intl. Conf. On Advances in Applied Science and Environmental Engineering - ASEE 2016 Copyright (C) Institute of Research Engineers and Doctors, USA .All rights reserved.

ISBN: 978-1-63248-086-6 doi: 10.15224/ 978-1-63248-086-6-54

molecules. Some interleukin receptors such as IL-15R, IL-2R, IL-18R and IL12Rare coupled to the common gamma chain and are involved in NK cell development and effector activity [16]. NK cells need priming by different factors, such as IL-15 secreted by dendritic cells(DC) [17] or macrophages [18], IL12 [18] [19] or IL-18[20], to act as a functional effector. In mammals, NK cell receptors based on their effects on cytotoxic activity are grouped into two major groups, inhibitory and stimulating receptors. All inhibitory NK cell receptors consist of one or more copies of immune receptor tyrosine based inhibitory motif (ITIM) in their cytoplasmic domains [21]. In contrast, several activating NK receptors employ adapter proteins including an immune receptor tyrosine based activation motif to pass on their signals. Structurally, NK cell receptors are also classified into two main groups: one group called NK C type lectin receptor consisting of a dimeric type II transmembrane domain, a domain very similar to $\mathrm{C}$ type lectin, and the other group named NK immunoglobulin (Ig) like receptors (KIR) having a type I transmembrane domain and Ig like domains [22] [23]. Members of the CLEC2 subfamily as ligands for NKRP1 subfamily resemble $\mathrm{C}$ type lectin like receptors (left). Ligands of CD94/NKG2 receptors and Ly49 receptors (mouse only) are MHC class I complexes consisting of a heavy chain, $\beta 2$ microglobulin, and a peptide (right). Symbols "+” and "_" show activating and inhibitory functions, respectively. For Nkrp1a, Nkrp1f, and Nkrp1g, functional activity remains unknown [24].
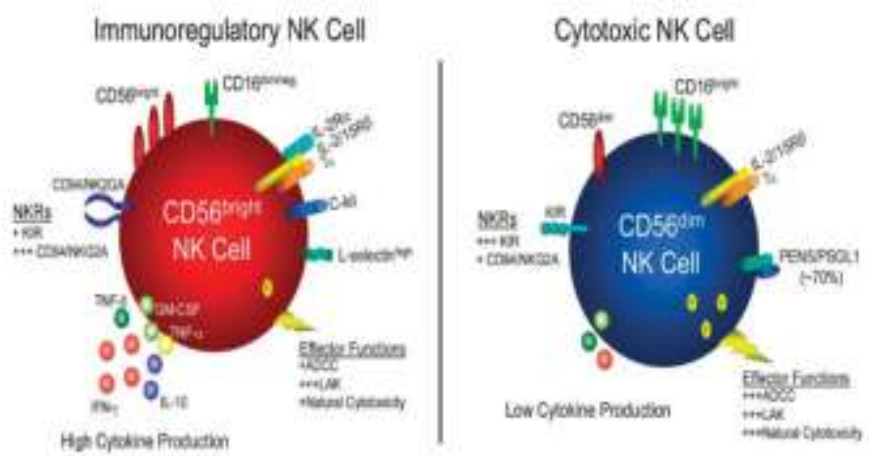

Figure 2. Human NK cell subsets. Following monokine stimulation the CD56 $6^{\text {bright }} \mathrm{NK}$ cells produce high levels of cytokines. This subset is an immune regulatory cell whereas CD56 ${ }^{\mathrm{dim}}$ has cytotoxicity activity (Cooper, et al., 2001; Farag \& Caligiuri, 2006).

Many of the activating or co activating NK cell receptors expressed by rat and human NK cells recognize self-antigens (Table 1). For example, the activatingNKG2D receptor recognizes numerous self-ligands in the host. Several of the activating Killer Cell Immunoglobulin like Receptor (KIR), Ly49, and CD94-NKG2C receptors are capable of recognizing self-major histocompatibility complex (MHC) class I proteins, and members of the "natural cytotoxicity receptors" group (such as NKp30, NKp44, and NKp46) (such as NKp30, NKp44, and NKp46) appear able to bind as yet undefined selfligands in the host [25] [26]. Thus NK cells express many activating receptors for self that could potentially drive auto reactivity [27]
Table 1. Activating and Co activating NK Cell Receptors (Orr \& Lanier, 2010).

\begin{tabular}{llll}
\hline Common Name & Ligand & Gene & Species \\
\hline Ly49D & $\begin{array}{l}\text { H-2D Hamster } \\
\text { MHC class I }\end{array}$ & Klra4 & Mouse \\
\hline Ly49H & MCMV-m157 & Klra8 & Mouse \\
\hline Ly49P & MCMV & Klra16 & Mouse \\
\hline NK1.1, NKR- & Unknown & Klrb1c & Mouse \\
P1C & & & \\
\hline NKR-P1F & Clrg & Klrb1f & Mouse \\
\hline PILR $\beta$ & O-glycosylation & Pilrb1 & Mouse \\
& CD99 & & Human \\
\hline 2B4 & CD48 & CD244 & Mouse, human \\
\hline LFA-1, CD11a & ICAM-1, 2, 3 & ITGAL & Mouse, human \\
\hline $\begin{array}{l}\text { CD94-NKG2C, } \\
\text { E }\end{array}$ & Mouse Qa-1 & KLRD1-KLRC2, & \\
& Human HLA-E & 3 & \\
\hline
\end{tabular}

\section{METHODOLOGY}

In this project it was intended to process the transfer of protein synthesis system of natural killer cells in to the hematopoietic stem cells harvested from the bone marrow of thigh bone of the experimental animal.to fallow the process and approach the goal it started with the separation of NK cells from the blood of experimental animal by LS column (magnet separation)[30][31], technique the blood cell are marked with CD49- and transfer to the columns with CD49+ after expose of the blood only the marked cells stick to the side of the column and they would be population of NK cells .After harvesting of NK cells from the columns it is subjected to flow cytometry test to ensure the population of NK cells is at least $80 \%$ of the harvested cells. After that the NK cells was cultured in animal cell culture medium for growth and increase of their population .In the culture we used NK cells receivers activator such as IL 12,15,18.In this particular experiment we used IL 12 to activate the growth of the cells and push their transgenic factor of their regulation to help to grow more and more for even better growth we used the help of Inomycin /PMA $10 \mathrm{mg}[28]$ and 50mg[29] for $24 \mathrm{hr}$ to provoke of growth of the NK cells and their ligands.After getting enough NK cells we extracted RNA by following the protocol of extraction of mRNA.After the extraction we used flow cytometry to ensure the concentration, purity and integrity of the extracted RNA is more than $80 \%$. with the help of Q PCR and appropriate designed primer we separated three target genes (Klra4, Klra8, KLRD1). In the other hand the stem cells was harvested from bone marrow of the rat and been cultured in to the stem cells special media (xeno- free expansion cell culture with SCF,FLt-ligands IL3,6) to prevent maturation the lipofectamine was inserted in the medium of the extracted RNAs and stabilized with the RNA to ready for transfer. After that the compound was transferred to the medium of stem cells for the last process, to transfer the protein synthesis system of NK cell's genes to the 
Proc. of The Fifth Intl. Conf. On Advances in Applied Science and Environmental Engineering - ASEE 2016 Copyright (C) Institute of Research Engineers and Doctors, USA .All rights reserved.

ISBN: 978-1-63248-086-6 doi: 10.15224/ 978-1-63248-086-6-54

hematopoietic cells. after the transfer, stem cells were maturated to NK cells with the possibility of faster division rate as NK cells and increase in population as NK cells against cancer cells with the help of transgenic NK cells.

\section{RESULTS AND CONCLUSION}

In zinc transporter lab at the faculty of science of university Malaya we designed a project to use all these information to create transgenic NK cells. The main objective of this experiment was to transfer the mRNA (extracted from natural killer cells of rats peripheral blood) of specific genes to hematopoietic stem cells (harvested from the thigh bone) of the experimental rat in other words the experiment was about to transferring the protein synthesis system of NK cell ligand's genes to HPSc cells to make cloned blood stem cells.to check the results of cloning procedures under the topic of transfection efficiency we used three techniques of flow cytometry, western blotting and FACS with help of green fb proteins .after adding the vector +medium to HSCs medium there was time required that the vector transfer the RNA to the cells ,in time table of $1 \mathrm{hr}, 24 \mathrm{hr}, 48 \mathrm{hr}$ and 7 days .within this period of time we checked the transfection efficiency separately by help of flow cytometry and FACS to observe the percentage of transfection .all the data is analysed by SPICE software that is designed most likely for flow cytometry results figure 3 shows the flow cytometry results of the transfection efficiency of the period of time $1 \mathrm{hr}, 24 \mathrm{hr}, 48 \mathrm{hr}$ .the results shows the percentage of transfected HSC cells.
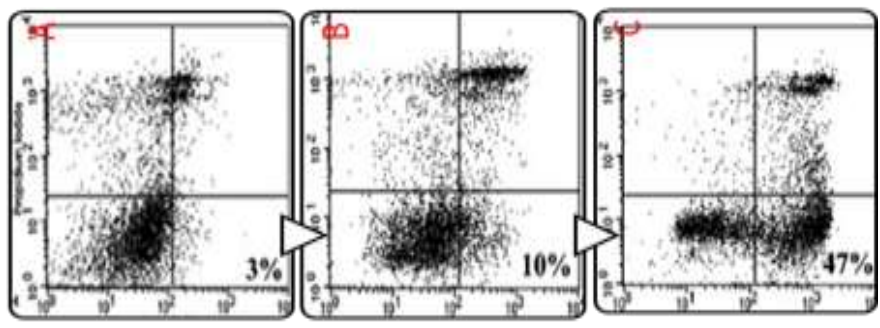

Figure 3. the results show that in the first hour(A) $3 \%$ of hematopoietic cells transformed to the NK cells and in 24 hour(B) $10 \%$ and in the 48 hour(C) $47 \%$ of stem cells transformed to the NK cells.

In fact, under the topic of transfection efficiency we used FACS method with the help of green fb (florescent protein) vector to check the percentage of transfected hematopoietic cells. figure 4 shows the printed results of the procedure in first hour and after 7 days.
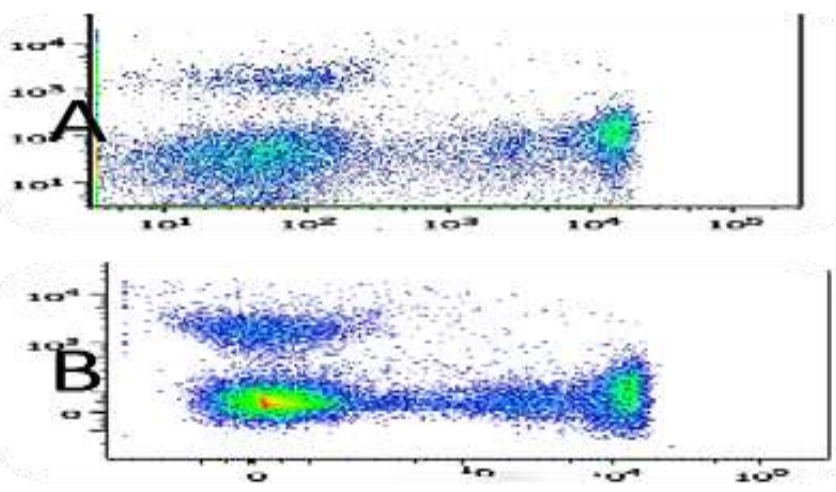

Figure 4: the first colour print(A) of FACS method shows the transfected stem cells and migration of the cells from right to left of the diagram and the second print (2) shows the increase of the transgene NK cells and migration of them to the right side of the diagram.

For the third and last transfection efficiency check of our medium we used western blotting protocol to reveal that the availability of RNA of NK cells in transfected stem cells. for that purpose, we ran the blotting gel to see the migration of RNA with the help of lipofectamin.

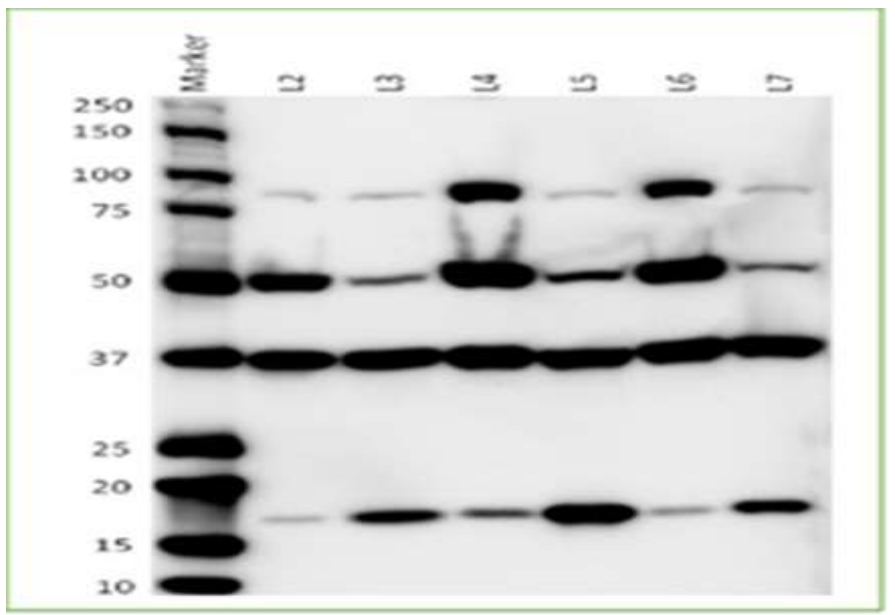

Figure 5: in western blotting that the gel shows the migration of the ly49d, ly49h and cd 94 genes throw the gel.

Based on our result to over view and analyse the relation of results in different protocols of transfection efficiency we used SPICE software designed specifically for the analysis of flow cytometry data. we transfer all data of the three different techniques in four different period of time $(1 \mathrm{hr}, 24 \mathrm{hr}, 48 \mathrm{hr}$ and 7 days) in diagram. The table 1 shows three different techniques data based on transfection percentage in period of time.

Table 1. three different technique of flow cytometry, western blotting and FACS show the transfection percentage of stem cells in $1 \mathrm{hr}, 24 \mathrm{hr}, 48 \mathrm{hrand} 7$ days.

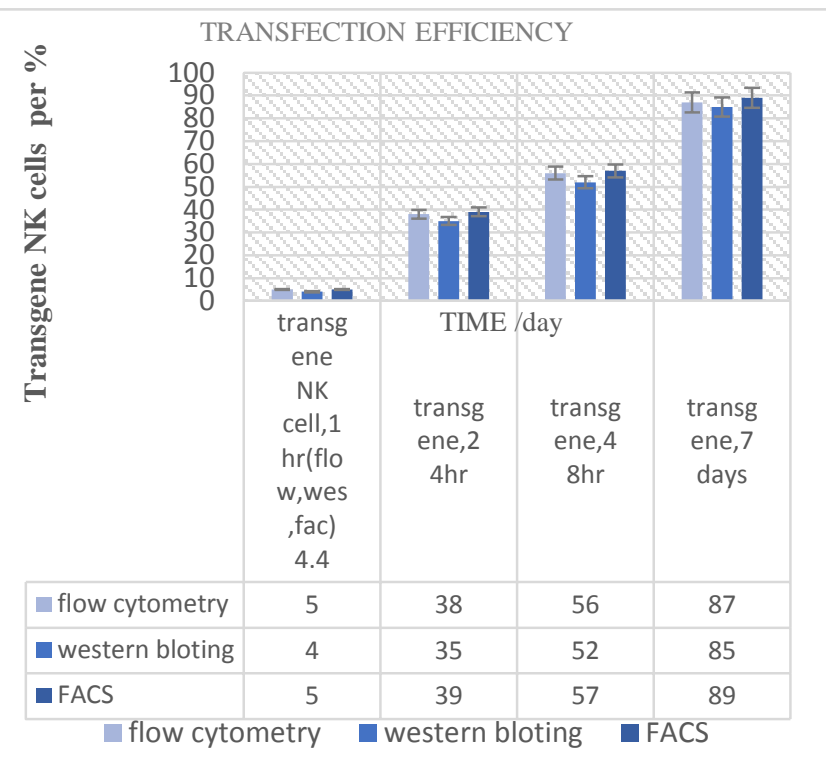


Based on our results after first hour of transferring medium + vector to our hematopoietic stem cells culture, flow cytometry shows $5 \%$ of stem cells transformed to the NK cells, western blotting shows $4 \%$ of stem cells transformed to the NK cell and FACS show 5\% of transfection. After that the $24 \mathrm{hr}$ present of medium + vector in the stem cell culture flow cytometry shows $38 \%$ transfection, western blotting shows $35 \%$ and FACS shows $39 \%$ of stem cells accepted the RNA from the NK cells. After 48 hours' flow cytometry shows $56 \%$ of transfection and western blotting shows $52 \%$ and FACS shows $57 \%$ of stem cells transformed to the NK cells.as it going based on our results after the period of 7 days our flow cytometry results shows $87 \%$ of our stem cells become NK cells and western blotting shows $85 \%$ and at last our results of FACS shows $89 \%$ of all hematopoietic stem cells genetically transformed to the NK cells. To understand the mean of all these data and to realize the total percentage of hematopoietic stem cells that transformed to the NK cells we calculate the total data mean and did draw the diagram of our all total percentages. Table 2 shows the diagram drown based on the means of total percentage of each period of time. based on our three techniques results the diagram shows that $4.6 \%$ of all our hematopoietic stem cells with in the period of 1-hour time transformed to NK cells. after 24 hours $33.3 \%$ and after 48 hours $55 \%$ and at the end after 7 days the mean of three used technique shows that $87 \%$ of all stem cells transformed genetically to NK cells.

Table 2. the diagram of averages of transfection efficiency of three different techniques.

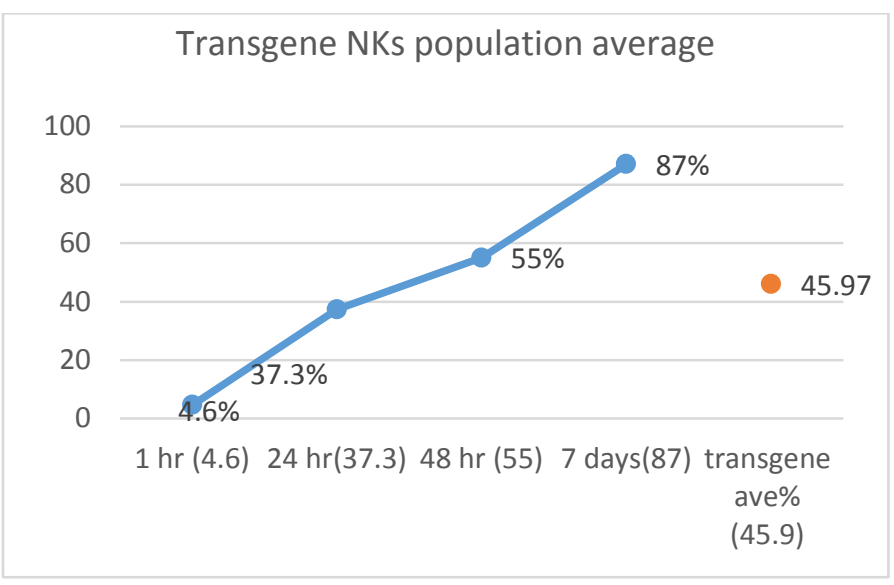

By analysing the data, we had by the SPICE drown this diagram that shows totally $45.97 \%$ of all hematopoietic stem cells transformed to natural killer cells. The transfection process could transform nearly half of the harvested stem cells to natural killer cells. On the other hand, the genes cloned inside the stem cells could produce the ligands, activator and inhibitory receptors on the cell membrane of stem cells.

\section{DISCUSION}

in zinc transporter lab of university Malaya, we designed a project to transform hematopoietic stem cells harvested from bone marrow of rat to natural killer cell to promote the immune system of the experimental animal against cancer. natural killer cells basically are the most prominent element of immune system against cancer cells but the only problem is that because the derive from hematopoietic cells as shown in figure 1 the quantity of them always less than cancer cells and they are not able to defeat cancer cells on their own that's why to cure cancer and against it we have to use procedures like chemotherapy, radiotherapy etc.so to help the body and promote the activity of the immune system against cancer we tried to transform the hematopoietic stem cells as the origin of NK cells to NK cells with regular dividing. That's why we design this project to extract RNA of NK cells and from hundreds of genes with help of Q PCR we separated three prominent gene Klra4, the gene what is responsible for activator ligand of LY94D and Klra8 as gene of another activator ligand $\mathrm{LY} 49 \mathrm{H}$ and KLRD1gene of co activating ligand of CD94.we cloned these three genes inside the stem cells with the help of a vector called lipofectamin and treat the medium with IL12,2, and 15 to help the transformed cells for coding the ligands on the cell membrane . after four domain of time as given in table 1 with the help of three techniques we checked the transfection efficiency and by data analysis software we conclude that $46 \%$ of all harvested stem cells that been emitted to vector + RNA genetically transformed to natural killer cells and mature stem cells dividing normally as an immune element. With help of this transformed NK cells and their ability to divide as normal cells that would be obviously faster than deriving from stem cells we would hope that by promoting ability of immune system of experimental animal could be a chance for cancer cure.

\section{V: REFRENCES:}

[1] Akira, S., Takeda, K., \& Kaisho, T. (2001). Toll-like receptors: critical proteins linking innate and acquired immunity. Nature immunology, 2(8), 675-680.

[2] Andoniou, C. E., van Dommelen, S. L., Voigt, V., Andrews, D. M., Brizard, G., Asselin-Paturel, C., . . . Degli-Esposti, M. A. (2005). Interaction between conventional dendritic cells and natural killer cells is integral to the activation of effective antiviral immunity. Nature immunology, 6(10), 1011-1019.

[3] Arase, H., Saito, T., Phillips, J. H., \& Lanier, L. L. (2001). Cutting edge: the mouse $\mathrm{NK}$ cell-associated antigen recognized by DX5 moncoclonal antibody is CD49b $(\alpha 2$ integrin, very late antigen-2). The Journal of Immunology, 167(3), 1141-1144.

Arstila, T. P., \& Lassila, O. (1993). Androgen-induced expression of the peripheral blood gamma delta $\mathrm{T}$ cell population in the chicken. The Journal of Immunology, 151(12), 6627-6633.

Avery, S., Rothwell, L., Degen, W. D., Schijns, V. E., Young, J., Kaufman, J., \& Kaiser, P. (2004). Characterization of the first nonmammalian T2 cytokine gene cluster: the cluster contains functional single-copy genes for IL-3, IL-4, IL-13, and GM-CSF, a gene for IL-5 that appears to be a pseudogene, and a gene encoding another cytokinelike transcript, KK34. Journal of interferon \& cytokine research, 24(10), 600-610.

[6] Bartel, Y., Bauer, B., \& Steinle, A. (2013). Modulation of NK cell function by genetically coupled C-type lectin-like receptor/ligand pairs encoded in the human natural killer gene complex. [Review]. Front Immunol, 4, 362. doi: 10.3389/fimmu.2013.00362

[7] Bartel, Y., Bauer, B., \& Steinle, A. (2013). Modulation of NK cell function by genetically coupled C-type lectin-like receptor/ligand pairs encoded in the human natural killer gene complex. Frontiers in immunology, 4. 
[8] Bayliss, C., Spies, U., Shaw, K., Peters, R., Papageorgiou, A., Müller, H., \& Boursnell, M. (1990). A comparison of the sequences of segment A of four infectious bursal disease virus strains and identification of a variable region in VP2. Journal of General Virology, 71(6), 1303-1312.

[9] Berg, T. P. V. D. (2000). Acute infectious bursal disease in poultry: a review. Avian Pathology, 29(3), 175-194.

[10] Biassoni, R., Pessino, A., Bottino, C., Pende, D., Moretta, L., \& Moretta, A. (1999). The murine homologue of the human NKp46, a triggering receptor involved in the induction of natural cytotoxicity. European journal of immunology, 29(3), 1014-1020.

[11] Böttcher, B., Kiselev, N., Stel'Mashchuk, V., Perevozchikova, N., Borisov, A., \& Crowther, R. (1997). Three-dimensional structure of infectious bursal disease virus determined by electron cryomicroscopy. Journal of virology, 71(1), 325-330.

[12] Bucy, R., Chen, C., Cihak, J., Lösch, U., \& Cooper, M. (1988). Avian $\mathrm{T}$ cells expressing gamma delta receptors localize in the splenic sinusoids and the intestinal epithelium. The Journal of Immunology, 141(7), 2200-2205.

[13] Bucy, R., Chen, C., \& Cooper, M. (1991). Analysis of gamma delta $T$ cells in the chicken. Paper presented at the Seminars in immunology.

[14] Bucy, R. P., Coltey, M., Chen, C. L. H., Char, D., Douarin, N. M. L., \& Cooper, M. D. (1989). Cytoplasmic CD3+ surface CD8+ lymphocytes develop as a thymusindependent lineage in chick-quail chimeras. European journal of immunology, 19(8), 1449-1455.

[15] Bumstead, N. (1998). Genomic mapping of resistance to Marek's disease. Avian Pathology, 27(S1), S78-S81.

[16] Bustamante, J., Boisson-Dupuis, S., Jouanguy, E., Picard, C., Puel, A., Abel, L., \& Casanova, J.-L. (2008). Novel primary immunodeficiencies revealed by the investigation of paediatric infectious diseases. Current opinion in immunology, 20(1), 39-48.

[17] Caligiuri, M. A. (2008). Human natural killer cells. Blood, 112(3), 461-469.

[18] Campbell, K. S., \& Purdy, A. K. (2011). Structure/function of human killer cell immunoglobulin-like receptors: lessons from polymorphisms, evolution, crystal structures and mutations. Immunology, 132(3), 315-325.

[19] Chaix, J., Tessmer, M. S., Hoebe, K., Fuséri, N., Ryffel, B., Dalod, M., . . . Vivier, E. (2008). Cutting edge: priming of NK cells by IL-18. The Journal of Immunology, 181(3), 1627-1631.

[20] Char, D., Sanchez, P., Chen, C., Bucy, R., \& Cooper, M. (1990). A third sublineage of avian T cells can be identified with a $\mathrm{T}$ cell receptor-3-specific antibody. The Journal of Immunology, 145(11), 3547-3555.

[21] Chiang, H.-I., Zhou, H., Raudsepp, T., Jesudhasan, P. R., \& Zhu, J. J. (2007). Chicken CD69 and CD94/NKG2-like genes in a chromosomal region syntenic to mammalian natural killer gene complex. Immunogenetics, 59(7), 603611.

[22] Confer, A., Springer, W., Shane, S., \& Donovan, J. (1981). Sequential mitogen stimulation of peripheral blood lymphocytes from chickens inoculated with infectious bursal disease virus. American journal of veterinary research, 42(12), 2109-2113.

[23] Cooper, M. A., Fehniger, T. A., \& Caligiuri, M. A. (2001). The biology of human natural killer-cell subsets. Trends in immunology, 22(11), 633-640.

[24] Cooper, M. A., Fehniger, T. A., Turner, S. C., Chen, K. S., Ghaheri, B. A., Ghayur, T., . . . Caligiuri, M. A. (2001). Human natural killer cells: a unique innate immunoregulatory role for the CD56bright subset. Blood, 97(10), 3146-3151.

[25] Coulibaly, F., Chevalier, C., Gutsche, I., Pous, J., Navaza, J., Bressanelli, S., . . . Rey, F. A. (2005). The birnavirus crystal structure reveals structural relationships among icosahedral viruses. Cell, 120(6), 761-772.
Cox-Singh, J., Hiu, J., Lucas, S. B., Divis, P. C., Zulkarnaen, M., Chandran, P., . . . Singh, B. (2010). Severe malaria-a case of fatal Plasmodium knowlesi infection with post-mortem findings. Malar J, 9(10).

[27] Daëron, M., Jaeger, S., Du Pasquier, L., \& Vivier, E. (2008). Immunoreceptor tyrosine-based inhibition motifs: a quest in the past and future. Immunological reviews, 224(1), 11-43.

[28] Davison, F., Kaspers, B., Schat, K. A., \& Kaiser, P. (2011). Avian immunology: Academic Press.

[29] De Maria, A., Biassoni, R., Fogli, M., Rizzi, M., Cantoni, C., Costa, P., . . . Cafaro, A. (2001). Identification, molecular cloning and functional characterization of NKp46 and NKp30 natural cytotoxicity receptors in Macaca fascicularis NK cells. European journal of immunology, 31(12), 3546-3556.

[30] Dennis, G., Kubagawa, H., \& Cooper, M. D. (2000). Paired Ig-like receptor homologs in birds and mammals share a common ancestor with mammalian $\mathrm{Fc}$ receptors. Proceedings of the National Academy of Sciences, 97(24), 13245-13250.

[31] Dohms, J., \& Jaeger, J. (1988). The effect of infectious bursal disease virus infection on local and systemic antibody responses following infection of 3-week-old broiler chickens. Avian Diseases, 632-640. 